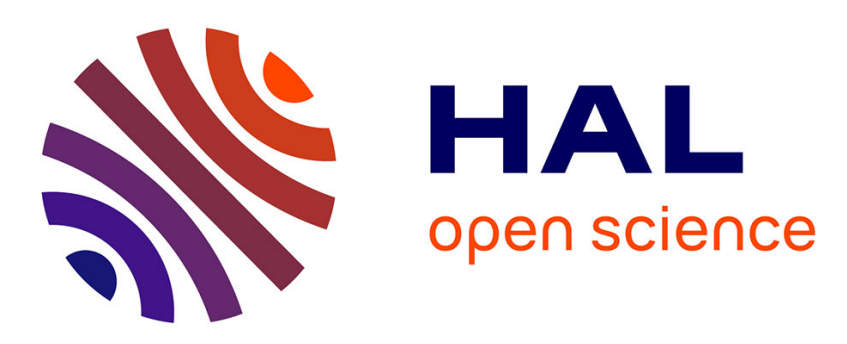

\title{
Traffic State Estimation Based on Eulerian and Lagrangian observations in a Mesoscopic Modeling Framework
}

Aurélien Duret, Yufei Yuan

\section{- To cite this version:}

Aurélien Duret, Yufei Yuan. Traffic State Estimation Based on Eulerian and Lagrangian observations in a Mesoscopic Modeling Framework. Transportation Research Part B: Methodological, 2017, 101, pp51-71. hal-01915206

\author{
HAL Id: hal-01915206 \\ https://hal.science/hal-01915206
}

Submitted on 14 Jun 2021

HAL is a multi-disciplinary open access archive for the deposit and dissemination of scientific research documents, whether they are published or not. The documents may come from teaching and research institutions in France or abroad, or from public or private research centers.
L'archive ouverte pluridisciplinaire $\mathbf{H A L}$, est destinée au dépôt et à la diffusion de documents scientifiques de niveau recherche, publiés ou non, émanant des établissements d'enseignement et de recherche français ou étrangers, des laboratoires publics ou privés. 


\title{
(C) 2018Manuscriptversion madeavailableunderCC-BY-NC-ND4.0license \\ https//creativecommons.org/licenses/by-nc-nd/4.0/
}

\section{Traffic State Estimation Based on Eulerian and Lagrangian observations in a Mesoscopic Modeling Framework}

\author{
Aurélien Duret ${ }^{\mathrm{a}}$, Yufei Yuan ${ }^{\mathrm{b}}$ \\ ${ }^{a}$ University of Lyon, ENTPE, IFSTTAR, LICIT, UMR-T9401, 25 avenue Francois \\ Mitterrand, 69675 Bron cedex \\ ${ }^{b}$ Faculty of Civil Engineering and Geosciences, Delft University of Technology, Stevinweg \\ 1, 2628CN, Delft, The Netherlands
}

\begin{abstract}
The paper proposes a model-based framework for estimating traffic states from Eulerian (loop) and/or Lagrangian (probe) data. Lagrangian-Space formulation of the LWR model adopted as the underlying traffic model provides suitable properties for receiving both Eulerian and Lagrangian external information. Three independent methods are proposed to address Eulerian data, Lagrangian data and the combination of both, respectively. These methods are defined in a consistent framework so as to be implemented simultaneously. The proposed framework has been verified on the synthetic data derived from the same underlying traffic flow model. Strength and weakness of both data sources are discussed. Next, the proposed framework has been applied to a freeway corridor. The validity has been tested using the data from a microscopic simulator, and the performance is satisfactory even for low rate of probe vehicles around $5 \%$.
\end{abstract}

Keywords: traffic state estimation, data assimilation, LWR model, mesoscopic model, Eulerian observation, loop data, Lagrangian observation, probe data, traffic monitoring, traffic forecasting,

Email addresses: aurelien.duret@ifsttar.fr (Aurélien Duret), y.yuan@tudelft.nl (Yufei Yuan) 


\section{Introduction}

\subsection{State of the art}

Traffic state estimation (TSE) is crucial in real-time dynamic traffic management and information applications. The essence of TSE is to reproduce traffic conditions based on available observation data. One class of available estimation methods does not make use of traffic flow dynamics, but relies on basic statistics and interpolation. These are referred to as data-driven methods. Another class of estimation methods relies on dynamic traffic flow models. These are referred to as model-based methods. The focus of this article is on the latter because it potentially provides better results than the former class in non-recurrent situations (work zones, accidents, social events, etc.), regarding the monitoring-forecasting capabilities.

Model-based TSE relies on two components : a model-based component and a data assimilation algorithm. The model-based component consists of two parts : $(i)$ a dynamic traffic flow model to predict the evolution of the state variables; and $(i i)$ a set of observation equations relating sensor observations to the system state. Thereafter, a data-assimilation technique is adopted to combine the model predictions with the sensor observations. For example, the Kalman filter (KF) $[20,3,14]$ and it advanced relatives, such as Extended KF [29], Unscented KF [22], Ensemble KF [30] have been widely applied in the field of traffic state estimation.

The same traffic flow model can be formulated in three two-dimensional coordinates regarding space $x$, time $t$ and vehicle number $n$. Laval and Leclercq [17] have presented three equivalent variational formulations of the first-order traffic flow models, namely $N(x, t)$ model, $X(t, n)$ model, $T(n, x)$ model respectively, under the theory of Hamilton-Jacobi partial differential equations. Under such defined coordinate systems, sensor observations from road networks can be defined into two categories : $(i)$ Eulerian sensing data - observations (e.g., aggregated speeds, flows) from spatially-fixed sensors (such as inductive loops, video sensors, and radar sensors) over a fixed report frequency, this type is dominating the information sources in the field of transportation research for decades; and ( $i i)$ Lagrangian sensing data information from probe samples at a fixed time interval (such as position and speed information of individual vehicles [14], and/or probe spacing and position information [25]), this class is becoming an increasingly popular source. In literature, most of TSE applications are based on the traditional space-time (Eulerian) formulation. Aggregated traffic quantities (e.g., flows, 
densities or speeds $[20,3,14,29,30])$ are usually considered as system states, but no individual vehicle tracking is involved. The popularity of this formulation is due to the fact that incorporating Eulerian data is straightforward and intuitive. Recent studies have shown that a first-order (LWR) traffic flow model $[19,24]$ can be formulated and solved more efficiently and accurately in vehicle number-time (Lagrangian-time) coordinates [18]. And its related Lagrangian formulation of TSE enables more accurate and efficient application of data assimilation methods, due to the solution to the mode-switching problem (traffic information travels in one direction), less non-linearity of the system model, and the nature set of observation equations to deal with Lagrangian data [31, 32]. However, the computation cost depends on the discretized platoon size (set to 1 vehicle classically) and time grid (often set around 1 second), which might be time consuming. The vehicle number space formulation inherits both the numerical benefits and modeling flexibility of the other two formulations.

Many previous studies have investigated the data assimilation problems on freeway networks with both Eulerian and Lagrangian observations under various modeling paradigms. Early studies in $[3,20]$ have applied the conservation law equations of traffic flow in Eulerian coordinates (LWR type [19, 24] and Payne's type [23], respectively) as the underlying traffic systems, to assimilate both simulated fixed-detector data and probe-vehicle data. Falling in this modeling category, the applications in $[30,14]$ have also performed data assimilation with isolated Eulerian and Lagrangian data. Work et al. [30] use a speed-based conservation law equation, while Herrera and Bayen [14] use an extended LWR model in the Eulerian formulation. Alternatively, the variational (HJ-PDE) formulation can be used as an equivalence to describe the same underlying traffic systems $[2,17]$. This modeling approach is considered to be much simpler to compute and numerically more accurate under same conditions, compared with the conservation law approach. However, only a few studies have applied such formulations for state estimation purposes. And the existing applications mainly adopt the aforementioned $N(x, t)$ model, where it considers the evolution of cumulated vehicle counts. For example, Claudel and Bayen [5] have applied the Hamilton-Jacobi formulation and generalized Lax-Hopf formula [2] for data assimilation and reconciliation utilizing loop and probe data; Newell's three-detector model [21] has also been extented for state estimation using heterogeneous data sources $[4,9]$ : loop detector data, Bluetooth travel time data and probe GPS data, respectively. 
To the best of our knowledge, none of previous research has applied the HJ-PDE formulation using the $X(t, n)$ model or the $T(n, x)$ model for traffic data assimilation. As discussed, in comparison to the $X(t, n)$ model, the simulation efficiency of the $T(n, x)$ model is independent on the discretized platoon size (set to 1 vehicle classically) and the spatio-temporal grid. Meanwhile, observation data are located along the vehicle number - space grids. Hence it is more advanced and convenient for data assimilation. This article presents a complete TSE framework for assimilating both Eulerian and Lagrangian data under a vehicle number - space (Lagrangian-space := L-S) formulation (namely, the $T(n, x)$ model). It inherits the classic modeling approach that has been widely applied for the data assimilation problem in the field of traffic management as well as meteorology, oceanography, image processing, etc. $[15,16,27]$. This modeling paradigm consists of system equations that capture the evolution of the state vector over time, and observation equations that capture the mapping of the state vector on the observations.

\subsection{Objectives and contributions}

This paper presents a generic data assimilation framework based on a mesoscopic-LWR model formulated in Lagrangian-space coordinates, using both Lagrangian and Eulerian observations. The term mesoscopic is in response to the two other counterparts, since the Lagrangian-time coordinates can apply in a microscopic simulation framework and the Eulerian coordinates can accommodate in a macroscopic one. In this work, the system model is the Lagrangian-space formulation of the LWR model. It individually represents vehicles but only tracks their states at cell boundaries. We will develop algorithms and observation models to incorporate data from both Eulerian and Lagrangian sensors, as well as considering the observation noise in both data sources. And we do not apply specific data assimilation techniques (e.g., KF-based approaches) ; instead we try to demonstrate the sequential data assimilation concepts via reasonable assumptions. The algorithms on how to estimate network traffic states under the proposed model-based framework from the two data sources will be the main contribution of this work.

\subsection{Contents of the paper}

This paper is organized as follows. Section 2 presents the underlying traffic dynamics, including its formulation, solutions and properties. Section 3 describes the methodology of the proposed TSE framework, including how to assimilate Eulerian data, Lagrangian data, and the combination of the two 
sources, respectively. Sections 4 and 5 illustrate the model validation and an application to a freeway corridor. Discussion and conclusions are drawn in Sections 6 and 7.

\section{LS-LWR model}

This section defines the underlying process model in the state estimation framework, where the model formulation, numerical solution and its properties are discussed.

\subsection{Conservation law and variational theory}

This section first presents a mesoscopic formulation of the LWR model as the process model in the estimation framework. The LWR model is formulated in vehicle platoon and space $(n, x)$ coordinates. The current mesoscopic formulation combines a vehicular description with macroscopic behavioral rules. It relaxes the temporal coordinate, and this entitles a transformation of a temporal progressing approach (e.g., in Eulerian or Lagrangian-time simulation framework) to an event-progressing approach (trigger event can be the change of time headway or pace, and/or a correction procedure based on an observation from fixed loops or probe vehicles).

The formulation follows the principle of the Hamilton-Jacobi (HJ) theory, to find an expression of the LWR model in Lagrangian-space coordinates. This model is also referred to as the $T$-model.

The LWR model can be described by a hyperbolic equation under the conservation law :

$$
\partial_{x} h-\partial_{N}(1 / V(h))=0
$$

Here, $h$ denotes the time headway. The inverse speed $1 / v$ (or called pace) can be derived from the fundamental diagram $1 / V(h)$.

Previous authors have proposed to apply variational theory in Eulerian coordinates $(x, t)[8]$ and Lagrangian coordinates $(n, t)[18]$. Here, we transpose the demonstration in Lagrangian-space coordinates $(n, x)$, following the same rationale in [18]. The problem can be expressed as the Hamilton-Jacobi derived from the fundamental diagram :

$$
\partial_{x} T=\frac{1}{V\left(\partial_{N} T\right)}
$$

Here, the function $1 / V$ represents the flux function of the problem. 

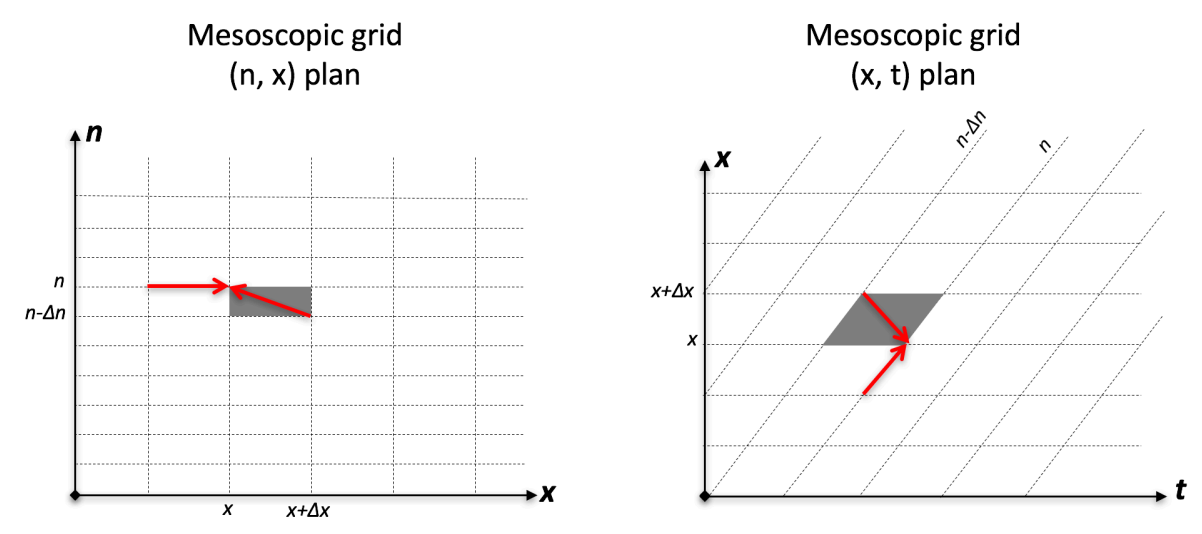

FiguRE 1: Numerical solutions in Lagrangian-space coordinates

\subsection{Numerical solutions in Lagrangian-space coordinates}

Here, a Godunov scheme [13] is applied to solve the conservation law equation (hyperbolic equation) above with an upwind method. This would preserve the numerical benefit of Lagrangian traffic flow models. Figure 1 illustrates the mesoscopic numerical grid (see grey area) for the Godunov scheme. On the mesoscopic grid, the time headway is determined by :

$$
h_{n}^{x+\Delta x}=h_{n}^{x}+\frac{\Delta x}{\Delta n} \cdot\left(\frac{1}{V\left(h_{n}^{x}\right)}-\frac{1}{V\left(h_{n-1}^{x}\right)}\right)
$$

The CFL condition that guarantees the convergence of the Godunov scheme $[6]$ is :

$$
\Delta n \geq \max _{h}\left|\partial_{h}\left(\frac{1}{V}\right)\right| \Delta x
$$

Alternatively, the problem can also be expressed in terms of $T(n, x)$ considering the 'passage time' flux that crosses the boundary of the cell $n$, regarded as a variational formulation of the $T$-model :

$$
\frac{T(n, x)-T(n, 0)}{\Delta x}=\frac{1}{V\left(\frac{T(n, x)-T(0, x)}{\Delta n}\right)}
$$

In this expression, $V$ depends on the fundamental diagram. Here, we consider a triangular fundamental with three parameters : the free-flow speed $v_{m}$, 
Fundamental diagram (Eulerian coordinates)

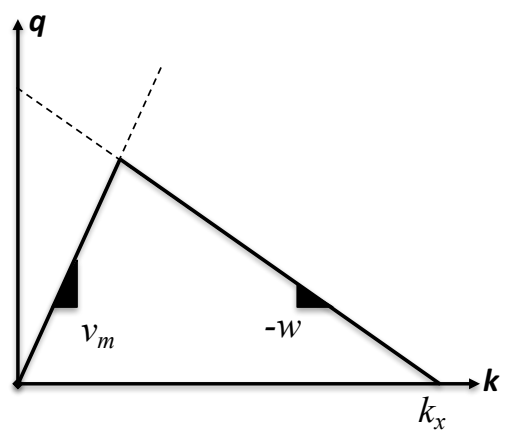

Fundamental diagram (Mesoscopic coordinates)

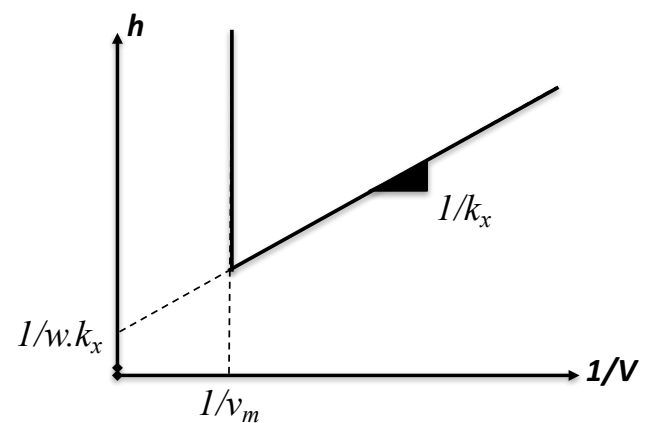

FiguRE 2: Fundamental diagrams in two coordinate systems 160

the maximum wave speed $w$ and the jam density $k_{x}$. Figure 2 represents the resulting fundamental diagram in Eulerian coordinates (left side) and in Lagrangian-space coordinates (right side). It can be expressed by :

$$
\frac{1}{V(h)}=\max \left(\frac{1}{v_{m}},-k_{x}\left(\frac{1}{w k_{x}}-h\right)\right)
$$

The numerical solution to the problem is simplified as [17] :

$$
T(n, x)=\max \left(T(n, 0)+\frac{x}{v_{m}}, T\left(0, x+\frac{n}{k_{x}}\right)+\frac{n}{w \cdot k_{x}}\right)
$$

Finally, the origin $n=0, x=0$ could be shifted to $n-\Delta n, x-\Delta x$ and we find:

$$
T(n, x)=\max \left(T^{d}, T^{s}\right),
$$


where $T^{d}=T(n, x-\Delta x)+\frac{\Delta x}{v_{m}}$ and $T^{s}=T\left(n-\Delta n, x-\frac{\Delta n}{k_{x}}\right)+\frac{\Delta n}{w \cdot k_{x}}$ represents the demand and the supply term, respectively. The demand term defines the arrival time of a vehicle from upstream in non-constrained (free-flowing) conditions. The resulting passage time of a vehicle is at least equal to its arrival time but could be delayed due to the downstream conditions. Thus, the supply time provides such information in constrained (congested) conditions.

This numerical solution indicates traffic flow is divided into vehicle platoons of certain size $\Delta n$, and road stretch is discretized into spatial cells of certain length $\Delta x$. Note that, the cell length $\Delta x$ in the simulation is not necessarily to be equal. The state in this formulation is the passage time $T(n, x)$ of vehicle platoons at cell boundaries. This state is always determined by the maximum of two uncorrelated terms : the demand (arrival) time and the supply time. For an elaborate description we refer to [17].

\subsection{Properties}

The current mesoscopic formulation is based on the notions from the variational theory. It can incorporate the numerical benefits and modeling flexibility of both Eulerian and Lagrangian-time models. Simultaneously, this formulation allows state distinction on both cell class and vehicle class, combining a vehicular description with macroscopic behavioral rules. It individually represents vehicles (platoons) but only tracks their passage times at cell boundaries. Therefore, travel times can be easily derived from the model, which is more convenient compared to other (e.g., Eulerian or Lagrangian) formulations of state estimation. This discrete model evolves state by state, with only one expression to consider all traffic conditions. Hence, it does not require memory and it is more flexible and time-efficient for data assimilation (no complex matrix inversion and multiplication). Moreover, the numerical scheme allows for long cells and cell boundaries can be located at network discontinuities only (merges, diverges, and lane-drops). In this way, the spatial discontinuities can address easily. The computation cost depends on the number of cell boundaries ( $x$-dimension) in the network and the number of vehicles ( $n$-dimension) to propagate during the simulation. Therefore, this would improve computational efficiency for large scale applications.

More importantly, this mesoscopic scheme is particularly convenient for data assimilation. In reality, the flow characteristics are mostly observed at fixed points (e.g., spatial fixed loop data) or along vehicle trajectories (e.g., vehicle-number fixed probe data). As discussed in literature that the Eulerian formulation is suitable for incorporating loop data and the Lagrangian-time 
formulation is suitable for probe data assimilation, the Lagrangian-space formulation is considered to be well-compatible for assimilating both types of observations. Because these observations are located on cell boundaries of the mesoscopic grid, which makes any traffic state estimation method convenient with this approach/formulation. This formulation can be easily coupled with any data assimilation techniques to perform state estimation. Due to the nature of the mesoscopic system model, the TSE might be not restricted to discretized mesoscopic $x-n$ grids. If we know any two boundaries in the network and an observation at a certain location or of a certain vehicle, we can generalize TSE for this specific assimilation problem.

\section{Data assimilation methodology}

Traffic flow prediction is an initial/boundary value problem, where a traffic model forecasts the evolution of traffic states on the network given initial/boundary states. Such data assimilation model-based approaches have been developed and widely applied in other fields, notably for atmospheric modeling and forecast $[11,27]$. Because predictions become more accurate when uncertainties of initial values are reduced, the data assimilation problem first combines observed data and the "first guess" provided by the model to estimate the mostly possible initial states at observation locations, also called analysis states. When the model is updated accordingly, it becomes ready to forecast the evolution of the system. [15] presents the typical data assimilation framework for atmospheric modeling (see chapter 5), where a sequential loop is run every time new data are observed. The composition of the loop returns an overarching theory of sequential data assimilation with four fundamental steps :

- Step 1 : the transformation operation makes observed data and model predictions comparable

- Step 2 : the global analysis provides the analysis states

- Step 3 : the model is updated accordingly

- Step 4 : the model forecasts the system evolution, by propagating information from data rich to data poor areas.

In the paper, methods for estimating traffic states based on loop and probe data are presented in sections 3.1 and 3.2, respectively. Then a method that combines both data sources is presented in section 3.3.

Three definitions with respect to different traffic states are given in the following : 
- an observation (o-) state is a traffic state measured by a sensor

- a background (b-) state is a state forecasted by a traffic flow model

- an analysis (a-) state is the result of an analysis procedure (or algorithm) that provides the most likely state regarding o- and b-states

\subsection{TSE based on loop data}

\subsubsection{Reminder on the existing methodology}

A data assimilation method using sole loop data first proposed in [12], has been validated on synthetic scenarios and tested on a large-scale network. Here the basic concepts are reviewed and the reader is referred to the paper for more details.

It requires the numerical scheme to be set as follows : $\Delta n$ to 1 and cells boundaries at each loop location. It considers flow and speed time series collected by loop sensors at locations $\left\{X_{\text {loop }}\right\}$ with a given frequency $\Delta T$. Then it is implemented as a sequential procedure, for which each sequence is divided into 4 successive steps (see Figure 3 ).

- Step 1: the o-state and b-state are collected and transformed

- Step 2: a Global Analysis is performed to (a-) state

- Step 3: the state of the model is updated accordingly, by adjusting arrival and supply times at cells boundaries

- Step4 : the model is run to provide a background state for the next sequence

As mentioned by the authors in [12], the update of the model has to be implemented so that the CFL stability condition is respected [6]. The solution proposed is a parsimonious adjustment of the demand and/or the supply terms at cell boundaries.

\subsubsection{Focus on step 3: Update of arrival and supply times at cell boundaries}

The a-state proposed by the Global Analysis consists of a regime $r^{a}$ and a headway $h^{a}$, at observation location and over the period P. $8\left(2^{3}\right)$ situations can be met (see Table 1) and the model needs to be updated accordingly.

The update consists of (i) adding, deleting, advancing or delaying vehicles at the cell boundaries and (ii) then updating the passing times of vehicles accordingly. In this paper, the update is identical to the one presented in [12] except that the passing times are managed slightly differently to be better suited for combining with the assimilation of probe data (see the next section) while keeping the same performance. 


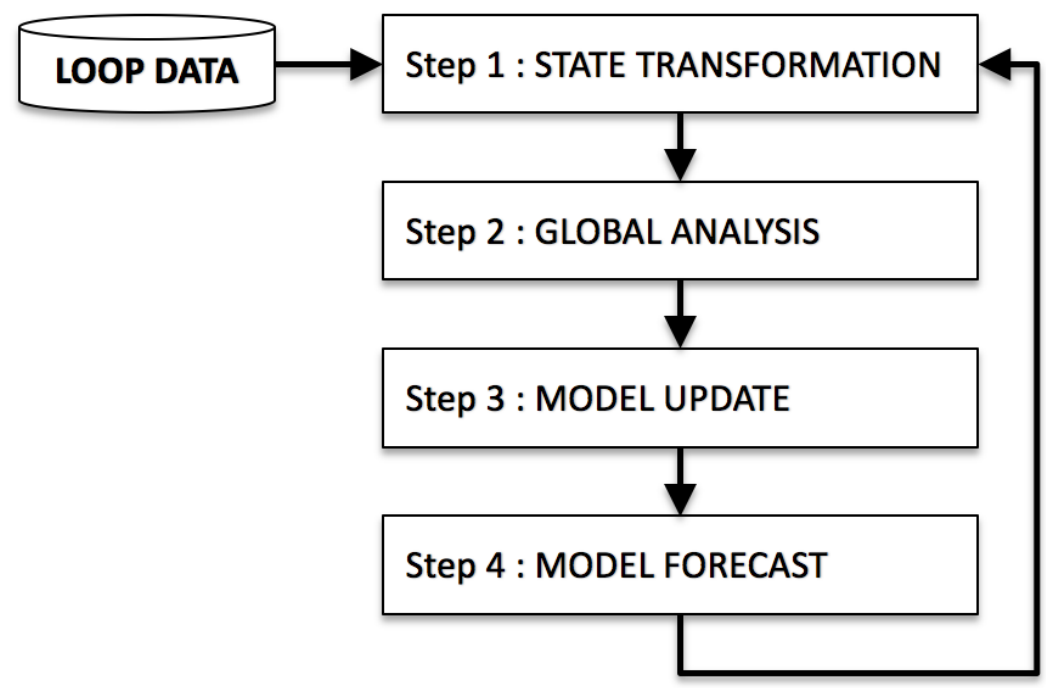

FIGURE 3: TSE based on loop data : methodology

TABLE 1: Summary of the 8 states combinations of a-state and b-state, source [12]

\begin{tabular}{|c|c|r|}
\hline & $r^{b}=0$ & $r^{b}=1$ \\
\hline$r^{a}=0$ & $h^{a}>h^{b} \ldots \ldots \ldots \ldots(1)$ & $h^{a}>h^{b} \ldots \ldots \ldots \ldots(5)$ \\
& $h^{a} \leq h^{b} \ldots \ldots \ldots \ldots(2)$ & $h^{a} \leq h^{b} \ldots \ldots \ldots \ldots(6)$ \\
\hline$r^{a}=1$ & $h^{a}>h^{b} \ldots \ldots \ldots(7)$ & $h^{a}>h^{b} \ldots \ldots \ldots \ldots(3)$ \\
& $h^{a} \leq h^{b} \ldots \ldots \ldots \ldots(8)$ & $h^{a} \leq h^{b} \ldots \ldots \ldots \ldots(4)$ \\
\hline
\end{tabular}

To do so the passing times are not updated directly. The method updates the demand and the supply terms at cell boundaries over the period $P$ If the analysis regime is free-flowing (combination 3, 4, and 7 in Table 1) :

$$
\left\{\begin{array}{l}
T^{s}(n, X)=-\infty \\
T^{d}(n, X)=T(n-1, X)+h^{a}
\end{array}\right.
$$

If the analysis regime is congested (combination 1,2 and 6 in Table 1) :

$$
\left\{\begin{array}{l}
T^{s}(n, X)=T(n-1, X)+h^{a} \\
T^{d}(n, X)=-\infty
\end{array}\right.
$$


Note that combinations 5 and 8 in Table 1 correspond to errors on both the local demand and supply. No update is required and the problem has to be addressed at global level (demand, model parameters, etc.).

\subsection{TSE based on probe data}

The data assimilation framework presented above is limited to Eulerian (loop) data while nowadays increasing amount of traffic data are collected by Lagrangian (probe) sensors. Thus a TSE estimator based on Lagrangian observations becomes essential for real applications. Probe sensors collect positions of equipped vehicles at a given time frequency. They are usually processed for providing aggregated indicators, for instance the mean speed per link. However, most of the wealth of probe data is lost during the aggregating process.

In this paper, the TSE estimator enables to assimilate positions and times without any aggregation process, which allow for using most of the details of probe data. The method is divided into 4 steps (see Figure 4).

- Step 1 : the o-state and the b-state are collected and transformed

- Step 2 : Global Analysis, which consists of estimating the $n$-index of probe vehicles

- Step 3 : the model is updated accordingly, which consists of adjusting arrival and supply times at cell boundaries of the model

- Step 4 : the model is run over the next sequence to provide a new background

The two following sections elaborate steps 2 and 3, which are the keys to successfully update traffic states.

\subsubsection{Focus on step 2: Estimating the $n$-index of probe vehicles}

Let us consider the probe vehicle $p$ that provides a set $S_{p}$ of observed timepositions denoted $\left\{t_{p, i}^{o}, x_{p, i}^{o}\right\}, i \in S_{p}$. Simultaneously the model provides a background state $T^{b}(n, x)$ at cell boundaries, from which analogous function $N^{b}(t, x)$ can be easily defined ( $T$ is a monotonically increasing function) upstream $\left(x=x_{u p}\right)$ and downstream $\left(x=x_{\text {down }}\right)$ probe positions. $N^{b}\left(t, x_{u p}\right)$ and $N^{b}\left(t, x_{\text {down }}\right)$ can then be considered for estimating local $n$-index of the probe based on variational principles applied for the three-detector problem $[7,8,21]$ as illustrated in Figure 5.

$$
n_{p, i}^{\text {local }}=\min \left(N_{u p, u}^{b}, N_{\text {down }, w}^{b}+k_{x} \cdot\left(x_{\text {down }}-x_{p, i}^{o}\right)\right)
$$




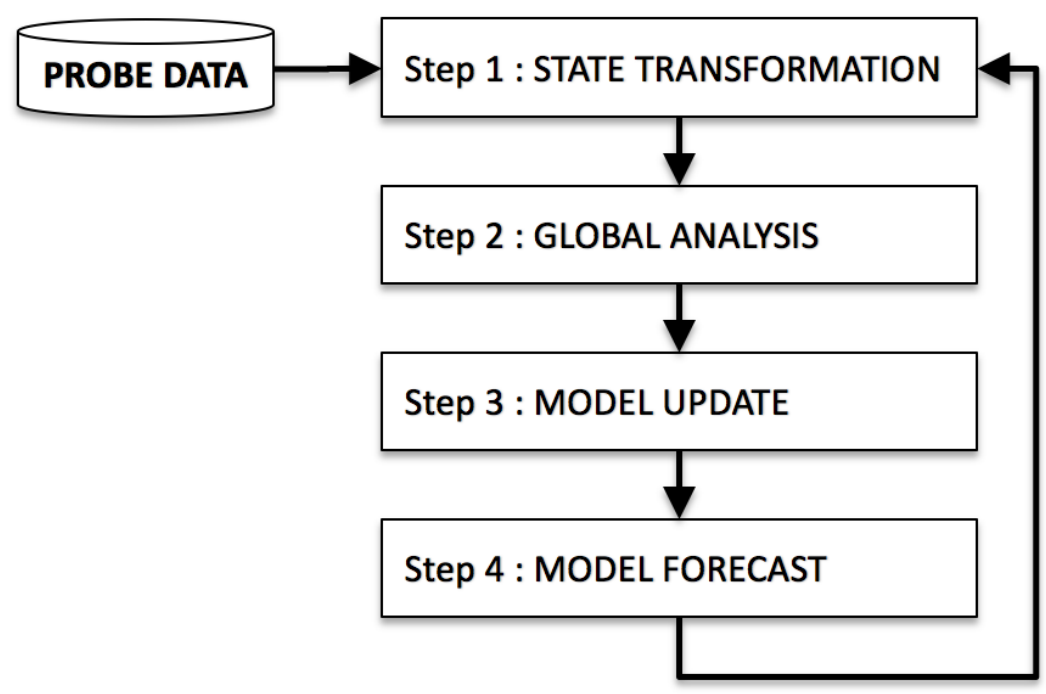

FIGURE 4: TSE based on probe data : methodology

305

306

$\left\{\begin{array}{c}N_{u p, u}^{b}=N^{b}\left(t_{p, i}^{o}-\frac{x_{p, i}^{o}-x_{u p}}{u}, x_{u p}\right) \\ N_{\text {down }, w}^{b}=N^{b}\left(t_{p, i}^{o}-\frac{x_{\text {down }}-x_{p, i}^{o}}{w}, x_{\text {down }}\right)\end{array}\right.$

Equation 11 provides the $n$-index estimated locally (for a single timeposition). At this stage, local $n$-index estimation could be flawed by four sources of errors : errors on the model parameters, errors on the boundary conditions, non-FIFO traffic conditions or occurrence of a traffic incident. Local errors on the estimated $n$-index may induce global inconsistencies on the resulting arrival/supply times. To tackle this problem, a global optimization is developed and it consists of two steps. The first step aims at building the variational proximity matrix, which returns the variational cost (in veh.) between each of the time-space observations from probes (with respect to the variational principles [8]). Based on that, the second step calculates the optimal $n$-index, denoted $n^{*}$. The optimal solution minimizes the entropy of the system $[1,28]$ while keeping a constant $n$-index along probe trajectories. The optimization procedure searches in the range of all possible $n$-indices, and this search range is defined by the minimum and maximum values from the variational principles and the range of local $n$-index estimation. The 


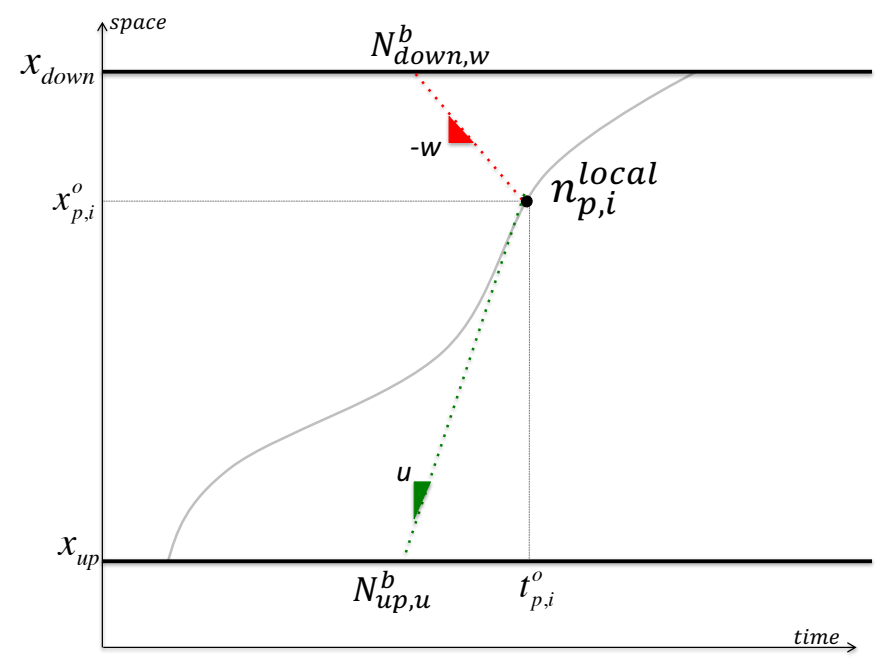

Figure 5: n-index estimation

entropy is defined according to [26] :

$$
E\left(n_{p}^{*}\right)=\sum_{i} \frac{n_{p, i}^{*}}{n_{p, i}^{\text {local }}} \ln \left(\frac{n_{p, i}^{*}}{n_{p, i}^{\text {local }}}\right)
$$

The final solution consists of the triplets $\left\{n_{p}^{*}, x_{p}^{o}, t_{p}^{o}\right\}$, where $n_{p}^{*}$ is the optimal $n$-index, and $t_{p}^{o}$ and $x_{p}^{o}$ are the observed time and position of the probe $p$.

\subsubsection{Focus on step 3 : Update of arrival and supply times at cell boundaries}

Once $a$-states are known, probe trajectories are considered as internal cell boundary conditions that are transformed into demand or supply conditions at neighboring cell boundaries. Here, we present the update of the arrival and the supply times at a cell boundary over a period $P$, considering that a set of probe vehicles has been analyzed.

Downstream : update of arrival times. The downstream cell boundary is influenced by probe vehicles located in a time window with a length $P$ and that moves with a free-flowing wave speed $u$, see Figure 6.

Within the influencing area, each probe vehicle provides information on its upcoming arrival times. When probe vehicles travel through a cell, successive 


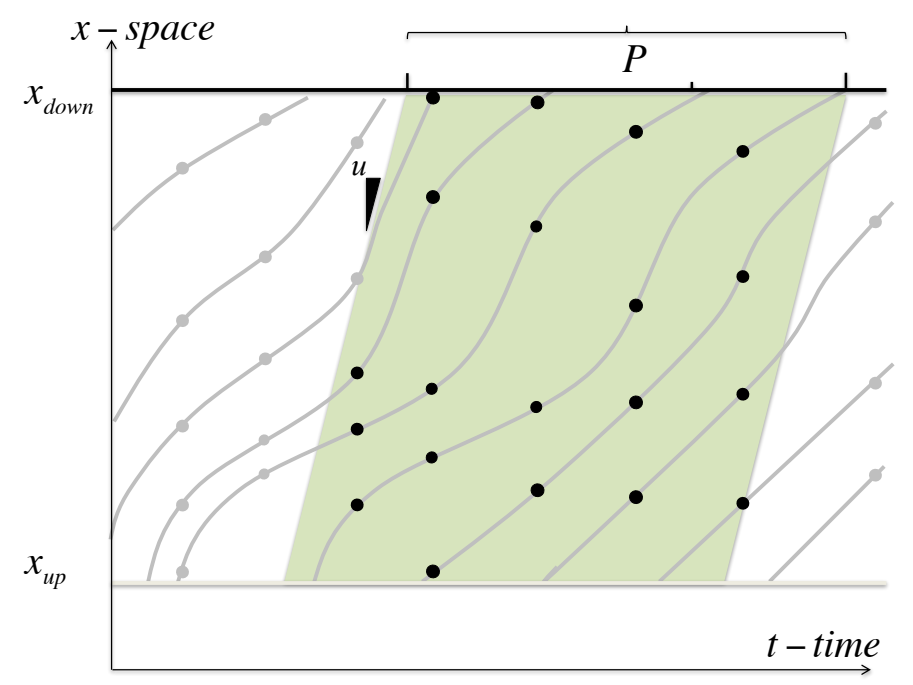

FiguRE 6: Oblique window for updating the demand term at the downstream cell boundary

time-positions provide feasible arrival times at the downstream cell boundary. For each probe vehicle, only the latest triplet $\left\{n_{p}^{a}, x_{p, i}^{o}, t_{p, i}^{o}\right\}$ is considered for updating the arrival time at the cell downstream, as illustrated in Figure 7.

$$
t_{a, n_{p}^{a}}^{a}=t_{p, i}^{o}+\frac{x_{\text {down }}-x_{p, i}^{o}}{u}
$$

Upstream : update of supply times. The upstream cell boundary is influenced by probe vehicles located in a time window with a length $P$ and that moves with a maximum jam speed $w$, see Figure 8 .

For each probe vehicle, triplets $\left\{n_{p, i}^{a}, x_{p, i}^{o}, t_{p, i}^{o}\right\}$ are considered as internal boundary conditions to revise supply times at the cell boundary upstream. Within the influencing area, the updated supply times respect as illustrated in Figure 9 :

$$
t_{s, n_{p}^{a}+\left(x_{p, i}^{o}-x_{u p}\right) \cdot k_{x}}^{a}=t_{p, i}^{o}+\frac{x_{p, i}^{o}-x_{u p}}{w}, \forall i \in S_{p}
$$

CFL condition The data assimilation process is sequential with time steps based on data time frequency $\Delta T$. The CFL stability condition has to be respected during the sequential update of the traffic model. It requires 


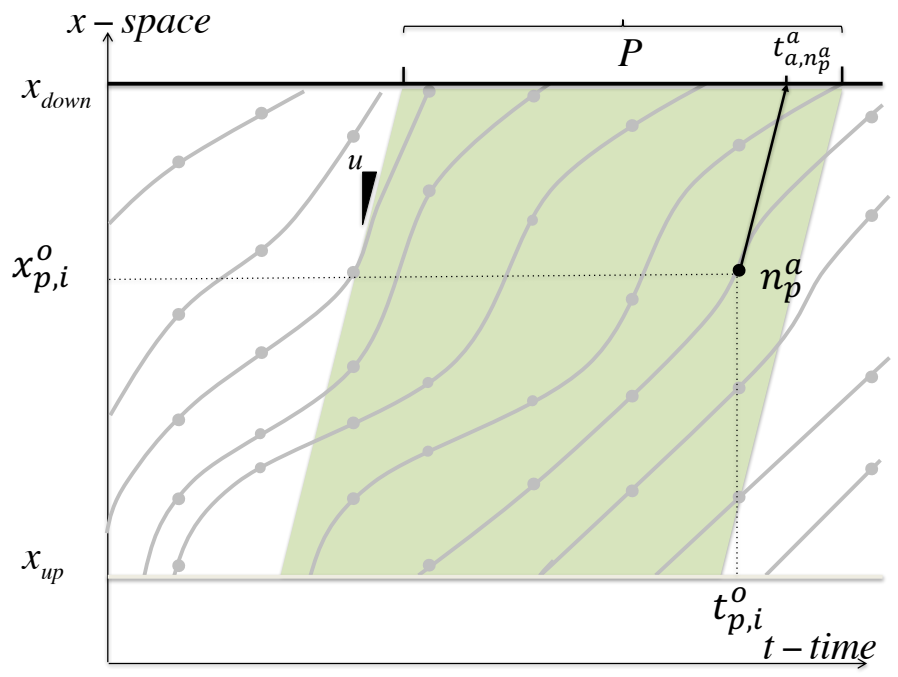

FiguRE 7: Update of the arrival time at the downstream cell boundary

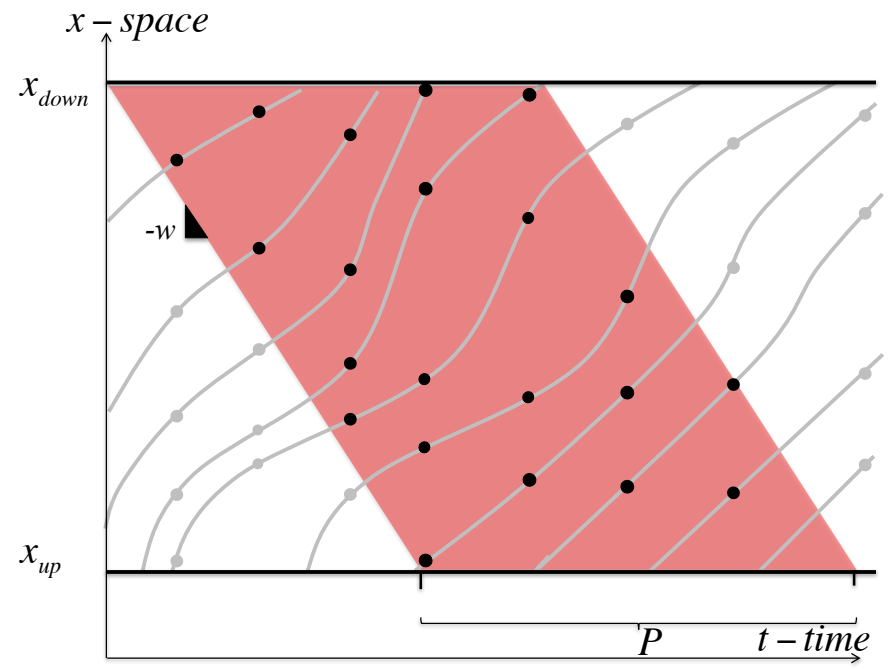

Figure 8: Oblique window for updating the supply term at the upstream cell boundary 


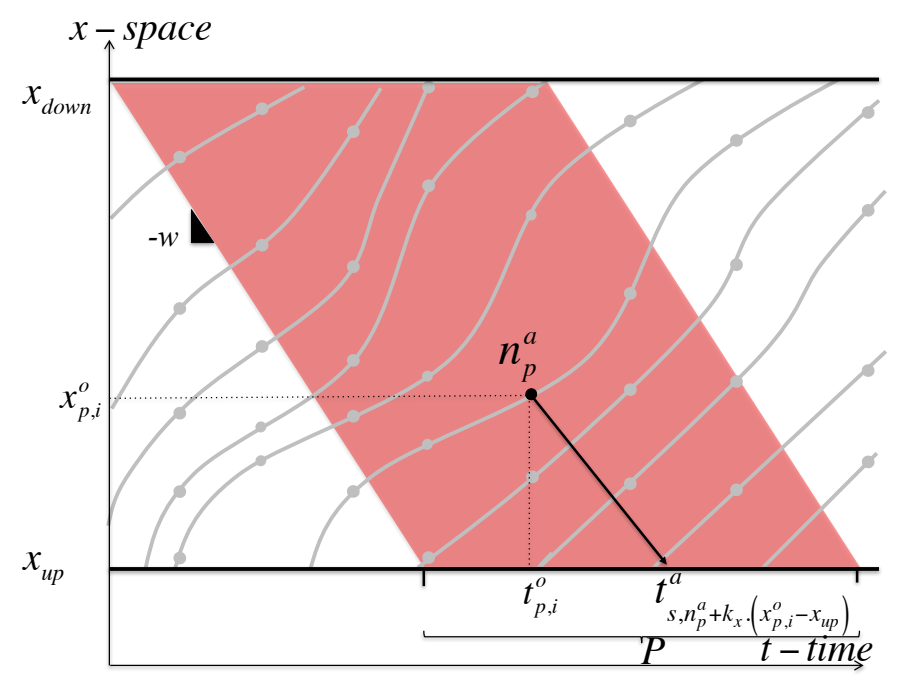

FiguRE 9: Update of the supply times at the upstream cell boundary

that each cell boundary has to be updated over a time period $\Delta T^{U}$, which is bounded as a wave cannot travel through a whole cell during this time period. Consequently, if $\Delta T \geq \Delta T^{U}$ then the updating process must proceed step by step (as described in the previous section) with a maximum time step $\Delta T^{U}$.

\subsection{Assimilating both loop and probe data}

Loop and probe data provide information of different nature, it is therefore impossible to fuse the two data sources to perform a one-shot assimilation process. Reviewing their respective actions, the two TSE estimators act in a complementary manner. On one hand, TSE based on loop data allows for an adjustment of the flow by adding - deleting - advancing - delaying vehicles at loop sensors locations. From a physical point of view, it acts as a 'flow regulator' at cell boundaries. On the other hand, the TSE based on probe data adjusts arrival and supply times at cell boundaries considering probe trajectories as internal cell-boundary conditions. From a physical point of view, it acts as a 'travel time regulator' along cells travelled by probe vehicles.

To make the best potential use of both data, we propose first to estimate trafic states from loop data at loop sensors locations and then to estimate traffic states from probe data everywhere else. The main reason for this se- 
quence is the following : TSE based on loop data improves the flow estimation at cell boundaries and therefore enhance the TSE based on probe data along cells. It results in a 7 steps methodology illustrated on Figure 10 :

- Step 1 : collection and transformation of the loop data and the model background states

- Step 2 : Global Analysis, which consists of estimating headway-regime pairs (a-states) at each loop location

- Step 3 : the model is updated accordingly (see section 3.1). At this stage, the updated model provides the best possible estimated traffic states at cell boundaries. This version of the model is considered as a new model background to be combined with probe data

- Step 4 : collection and transformation of the probe data and the (updated) model background

- Step 5: Global Analysis, which consists of estimation the n-index of probes along cells

- Step 6 : update of the model accordingly, by revising arrival and supply times at every cell boundaries, except those already updated during the step 3.

- Step 7 : run the model over the next sequence

Here again, this sequence has to be implemented respecting CFL stability condition mentioned in section 3.2.2.

\section{Model validation}

This section aims to analyze and validate the performance of the TSE methodology with loop sensors and probe sensors (separately and jointly).

\subsection{Experimental validation setup}

The ground truth is emulated based on a microscopic LWR model (Newell's car-following model [21], equivalent to the LWR model at a macroscopic scale). The model has been run on a homogeneous road stretch $(L=2000 \mathrm{~m}$, single lane) with a demand-supply scenario so that a congestion propagates through the network, see vehicle trajectories in Figure 11. A loop sensor located in the middle of the network $(x=1000 \mathrm{~m})$ collects flows and speeds with an aggregation period of 1-minute. Moreover, $10 \%$ of the vehicles are considered as probe sensors for which time-position information is reported at every $30 \mathrm{~s}$. 


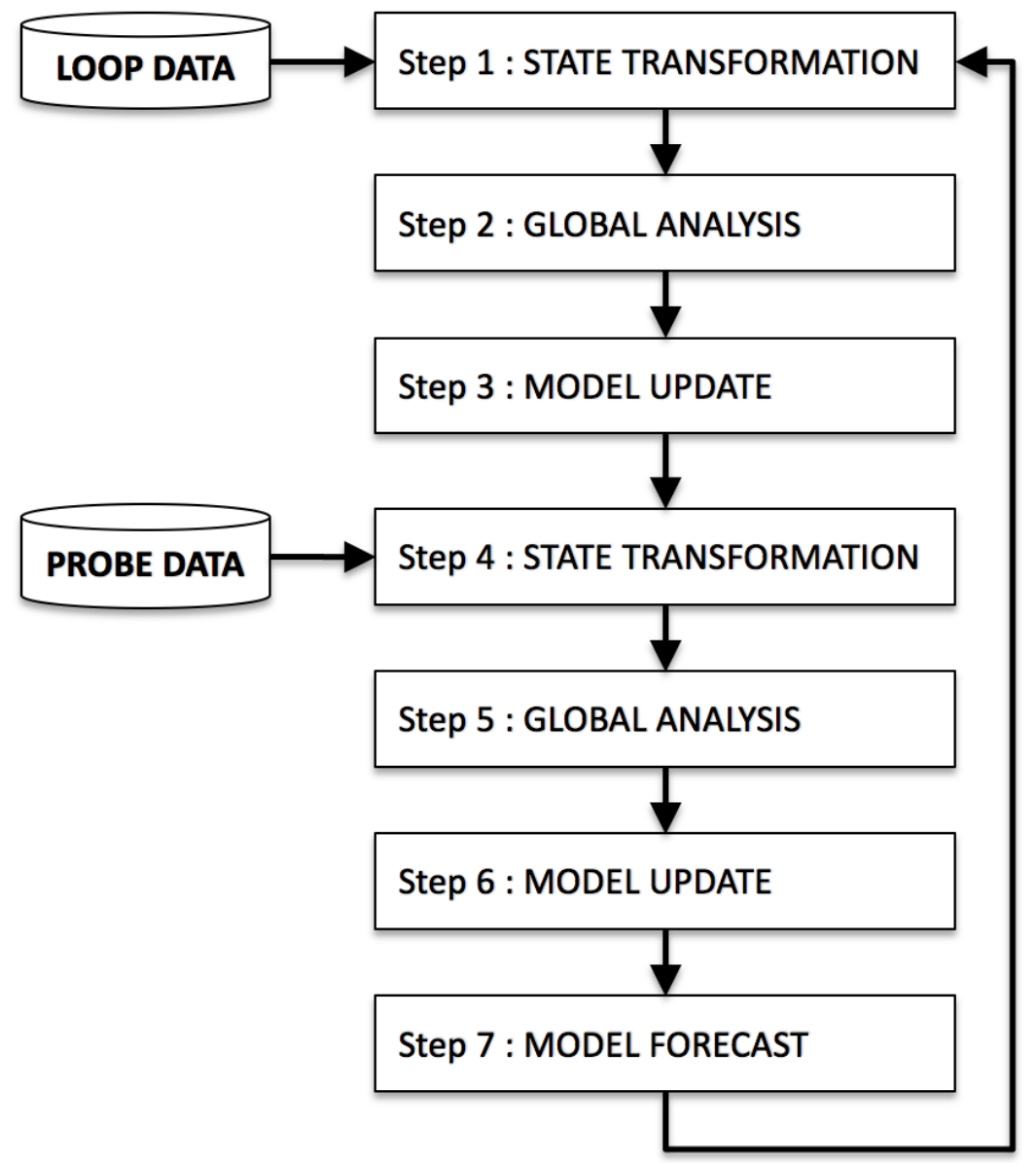

Figure 10: TSE based on loop and probe data : methodology 


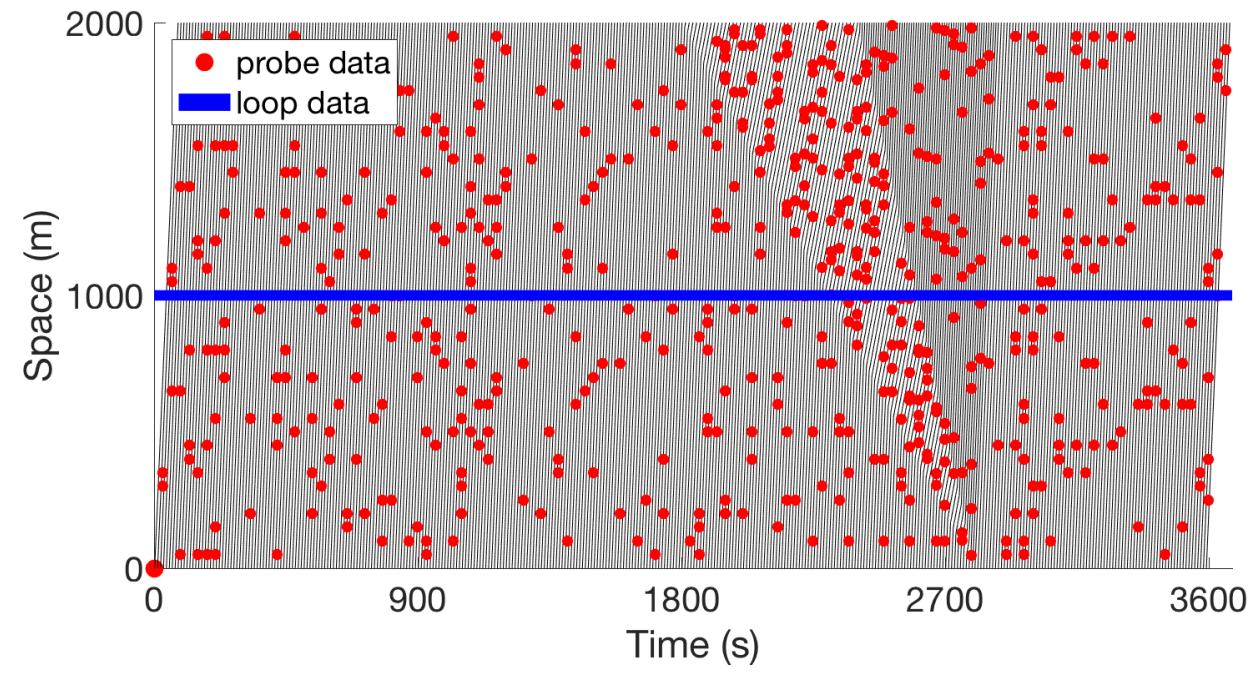

Figure 11: Observational model

The traffic flow model is a LS-LWR model. The network is composed of 2 cells of $1000 \mathrm{~m}$ in length, upstream and downstream of the loop sensor location (namely the upstream and the downstream cells). The demandsupply scenario has also been predefined with an approximative demand and a high supply so that traffic conditions are always free-flowing on the network.

\subsection{TSE based on loop observation model}

Figure 12 provides the estimated traffic states considering data from the loop sensor. In this figure, traffic states have been rearranged to provide travel times over the two cells. The red line provides the reference (ground truth) travel times and the blue line returns the reconstructed travel times.

Upstream cell. Until the time $t=2000 \mathrm{~s}$, the traffic conditions are freeflowing. Between the time period $t=2200 \mathrm{~s}$ and $t=2800 \mathrm{~s}$, a congestion propagates through the upstream cell. The estimated traffic states comply with the observed travel times, which validates the ability of the TSE estimator to adjust the network supply at the loop sensor location.

Downstream cell. Downstream the loop sensor, the estimated traffic states are free-flowing until the end of the simulation, whereas the ground truth indicate that a congestion occurs. Indeed, the loop sensor data only indicate 

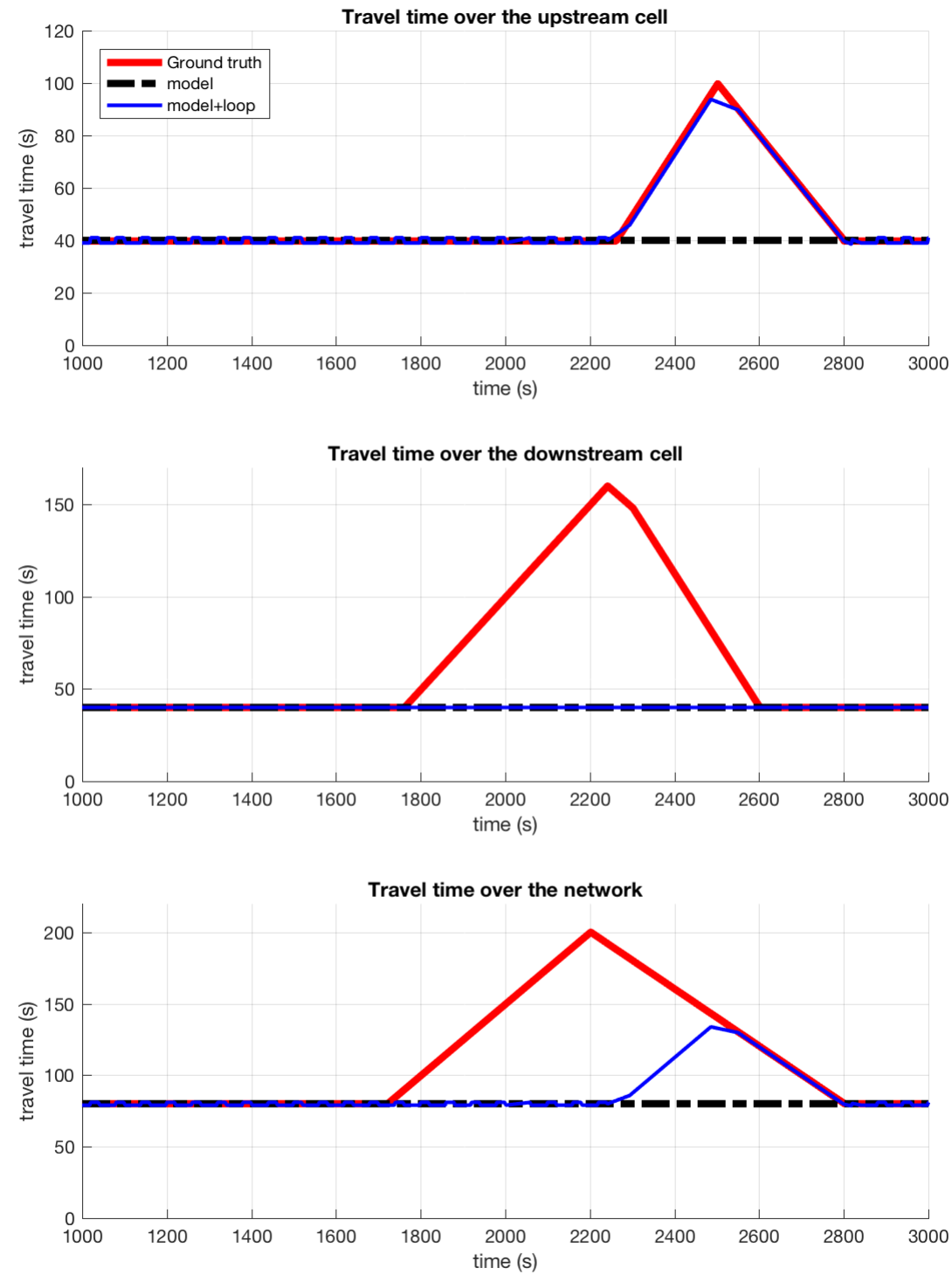

Figure 12: Travel time estimation from loop data assimilation 
a reduced congested flow at the cell boundary, however the traffic model is unable to propagate such information toward the downstream direction but only upstream direction.

In summary, when a congestion occurs, loop sensors can estimate travel times providing that congestion states have passed over the loops. The result shows that travel times might be underestimated over the network level. And this underestimation will become significant when traffic congestion is triggered far downstream the loop sensor. We conclude that for operational purposes loop sensors have to be located as close as the triggering location of a jam/bottleneck to provide accurate estimation. In addition, the complementary information from downstream loop sensors can improve the performance of data assimilation.

\subsection{TSE based on probe observation model}

Figure 13 provides the estimated traffic states considering probe data only. The performance of TSE based on sole probe data provides similar performance over the two cells. It is noteworthy that TSE is very responsive as the congestion phenomenon occurs, mainly due to the probe data with a homogeneous coverage of the network both in time and space. It should also be noted that travel times are underestimated in this validation scenario due to the experimental setup. The traffic model considers a low demand versus high supply scenario. Information from probes allows for an adjustment of the supply times at the intercell boundary, but it does not rectify the underestimated flow demand (from downstream) and thus underestimate travel times. Note that the result depends on the experimental setup as travel times will become overestimated if the demand from the upstream boundary is overestimated.

We conclude that for operational purposes, the knowledge of the demand at any point of the network is decisive and critical when probe data are used for estimating traffic states. The estimation can be enhanced with an accurate prior estimation of the demand ; or combining probe data with loop data, as proposed in section 3.3.

\subsection{TSE based on loop and probe observation model}

Figure 14 provides the estimated traffic states considering both loop and probe data. The results show the travel time estimation here outperforms and cumulates the benefits mentioned for loop and probe observation model considered separately. 

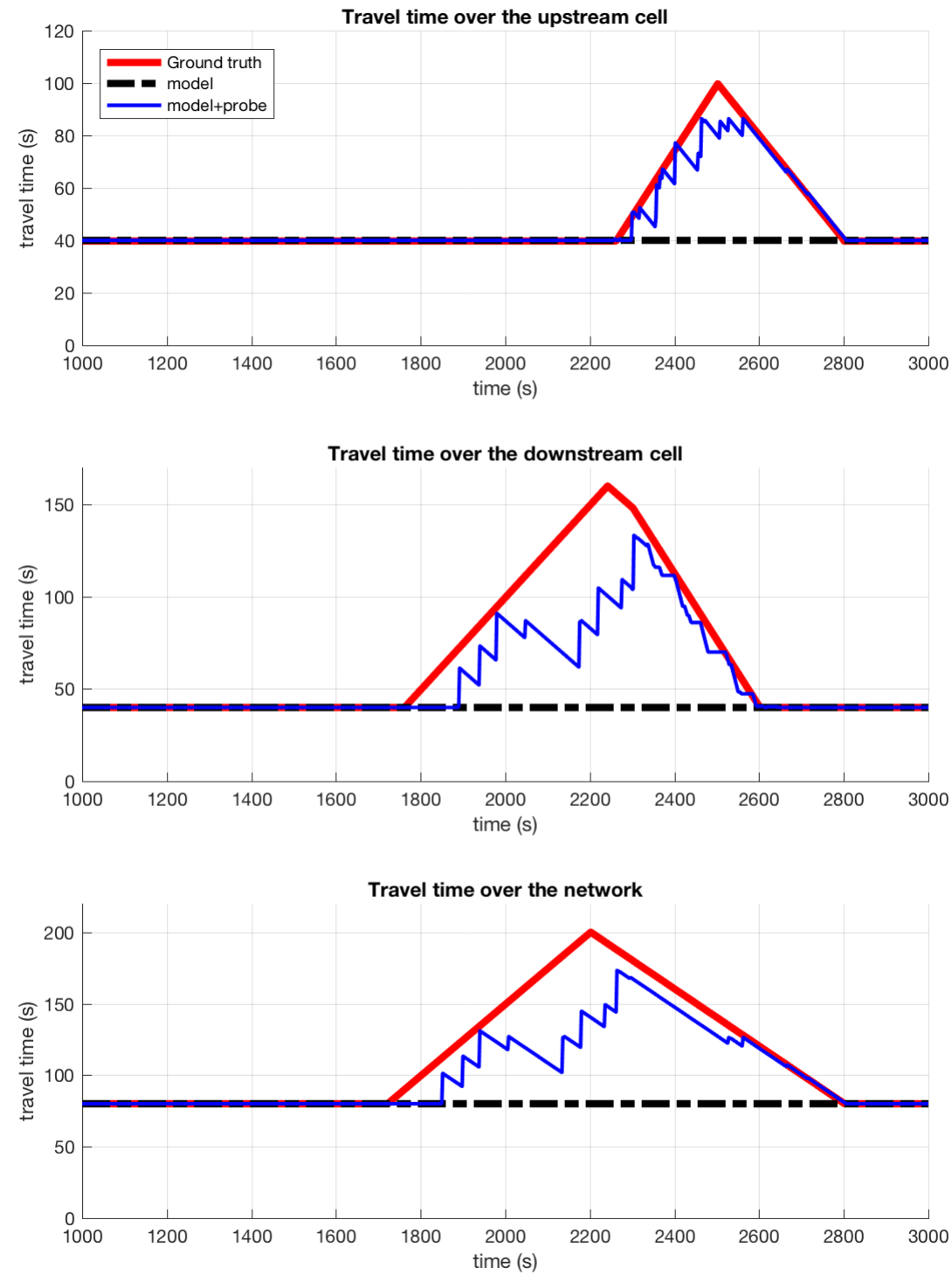

FiguRE 13: Travel time estimation from probe data assimilation 

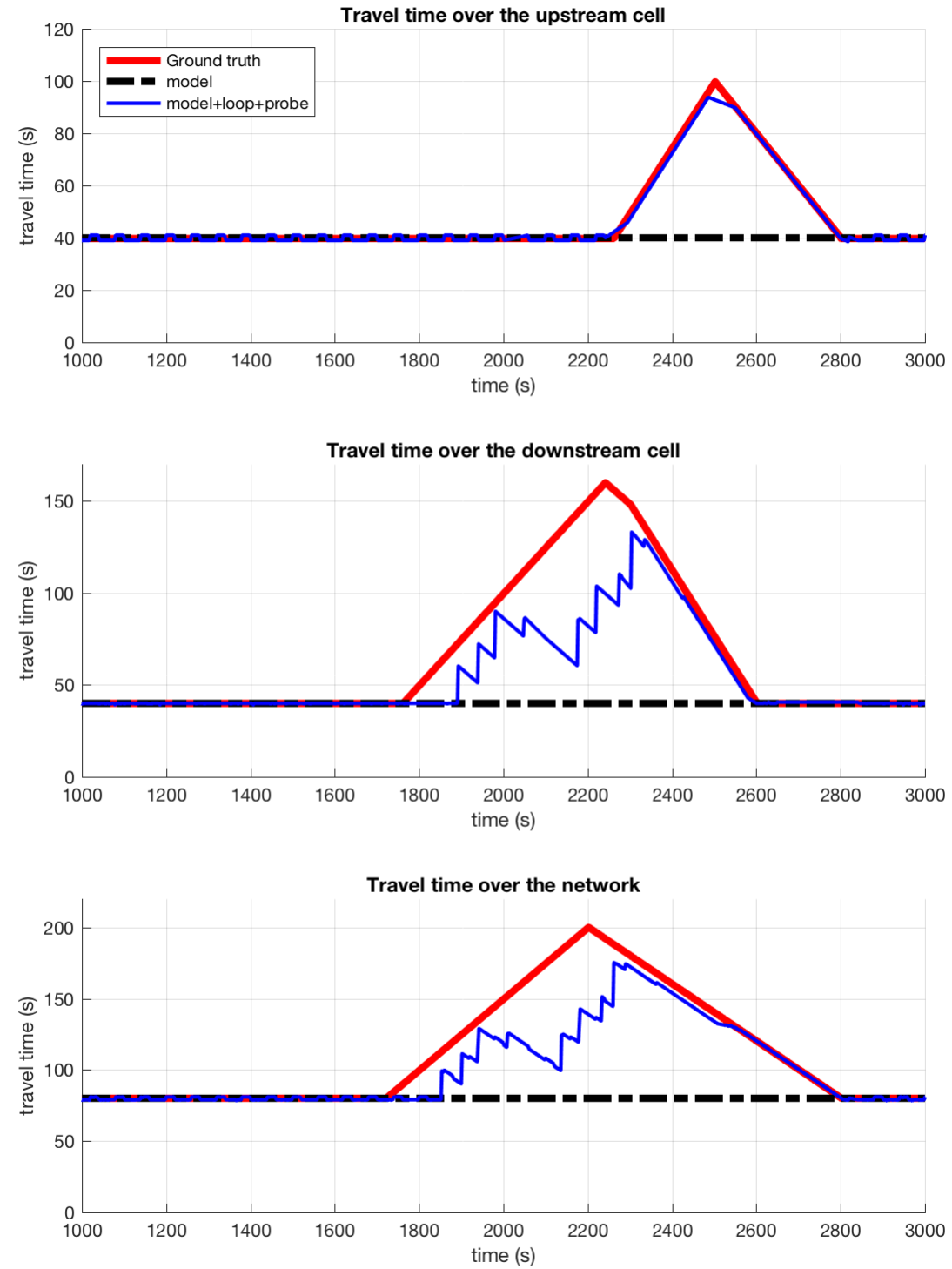

Figure 14: Travel time estimation from loop and probe data assimilation 
Upstream cell. The performance are identical to the those provided by the loop observation model. The travel time is properly estimated and fit the ground truth travel time.

Downstream cell. The performance is slightly enhanced compared to the results obtained with probe observations only. It confirms that both observations are very complementary when assimilated in the framework proposed in the paper.

Table 2 provides three Measurements of Effectiveness (MoEs) that have been calculated over the congested period $=[30-45] \mathrm{min}$ : root mean square error (RMSE), mean absolute percentage error (MAPE) and mean percentage error (MPE). All the MoEs globally confirm the previous comments.

TABLE 2: Scenario with a homogeneous stretch of road

\begin{tabular}{|l|c|c|c|c|}
\hline & model & model + loop & model + probe & model + (loop and probe) \\
\hline RMSE $(s)$ & 229 & 58 & 28 & 28 \\
\hline MAPE $(\%)$ & 34 & 25 & 12 & 11 \\
\hline MPE $(\%)$ & -34 & -25 & -12 & -11 \\
\hline
\end{tabular}

\section{Application to a freeway corridor}

The previous section demonstrates the exactness of the estimator when applying to a network with FIFO conditions and homogeneous driving behavior. These assumptions are restrictive and not reflective of reality. This section aims at evaluating the performance of the estimator considering a multi-lane corridor with on- and off-ramps, with a relaxed FIFO assumption and distributed driving behavior.

\subsection{Preparation of the observational model}

Ground truth data have been emulated based on a microscopic traffic simulator (FOSIM [10]). This simulator is developed at the Delft University of Technology, specially designed for the detailed analysis in freeway networks. All the parameters in terms of driving behaviors have been calibrated and validated based on data from Dutch freeways. A three-lane freeway with one on-ramp and one off-ramp is designed, as illustrated in Figure 15 (the first $500 \mathrm{~m}$ as the warming-up section in Simulation, the last $1000 \mathrm{~m}$ as the cooling-down section). 

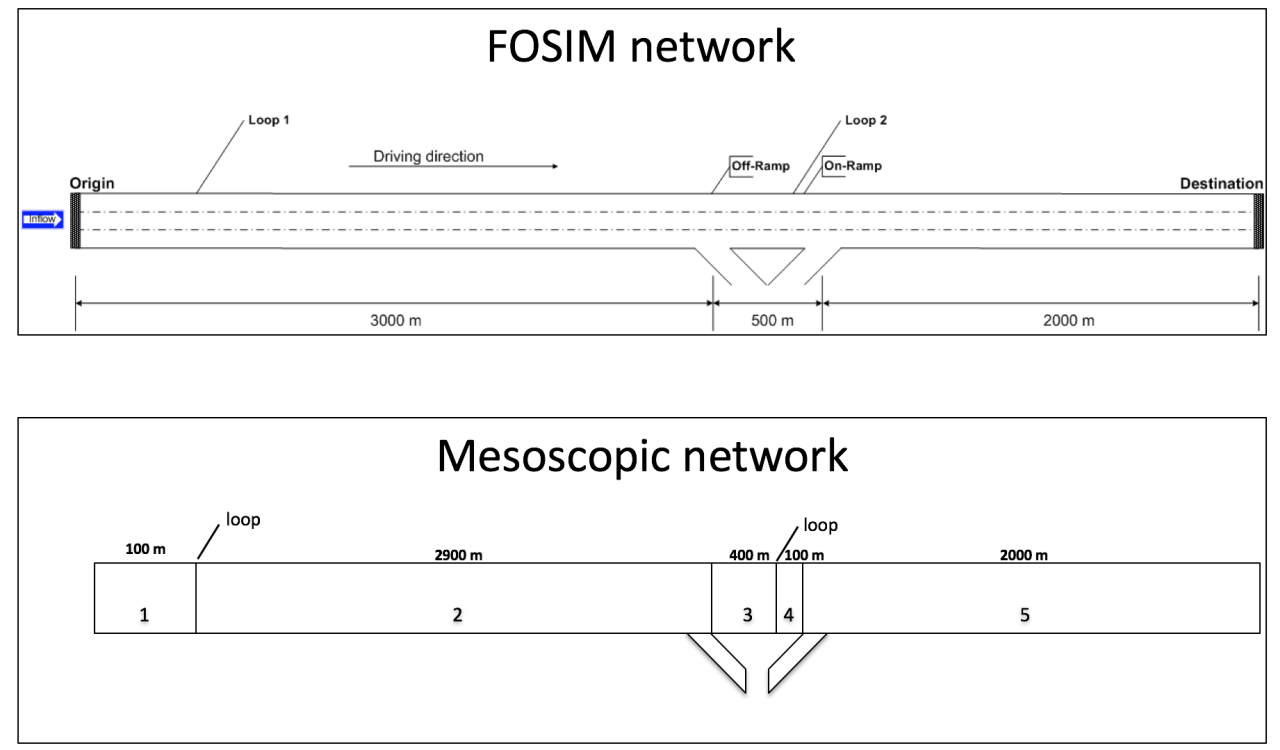

FiguRE 15: Networks

A demand-supply scenario has been built in such a way that a congestion is onset at the on-ramp. The model has been run twice : scenario 1 provides traffic with only passenger cars whereas scenario 2 considers a mixed traffic, with $90 \%$ cars, $10 \%$ trucks. The resulting time-space diagrams and travel times for the seed 1 are illustrated in Figure 16.

Based on FOSIM simulation results, Eulerian and Lagrangian observation models have been built. First, loop sensors have been located on the main road : loop $1-100 \mathrm{~m}$ after the entrance of the network, and loop $2-100 \mathrm{~m}$ upstream of on-ramp. Second, 10\% equipped probe vehicles return their exact positions every 20 s.

\subsection{Preparation of the traffic model}

The traffic model is the mesoscopic LWR model applied on a network with 7 cells : five cells for the main road (numbered from 1 to 5), one cell 6 for the off-ramp and one cell 7 for the on-ramp. Cell boundaries 1-2 and 3-4 are located at loop sensor locations. Boundary conditions (demand-supply) are supposed to be known approximatively and parameters of the mesoscopic LWR have been set with the following default values : $u=110 \mathrm{~km} / \mathrm{h}, w=18$ $\mathrm{km} / \mathrm{h}$ and $k_{x}=150 \mathrm{veh} / \mathrm{km} / \mathrm{lane}$. Results obtained from the underlying 


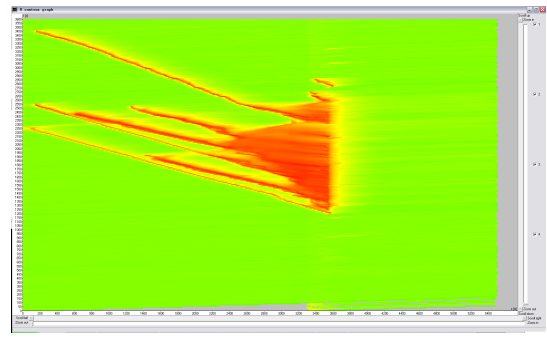

(a) time-space diagram

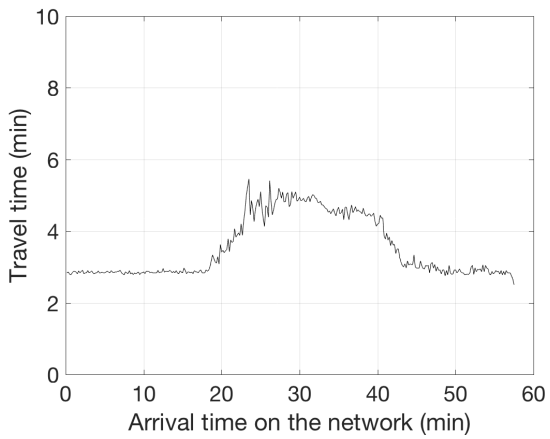

(c) travel times

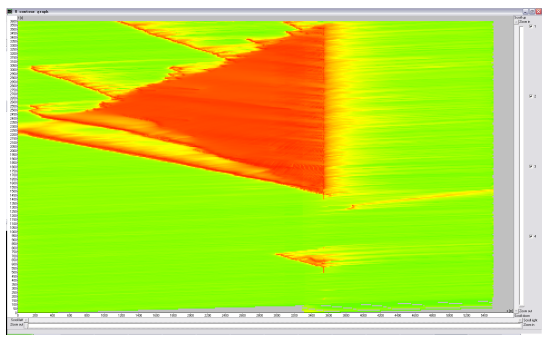

(b) time-space diagram

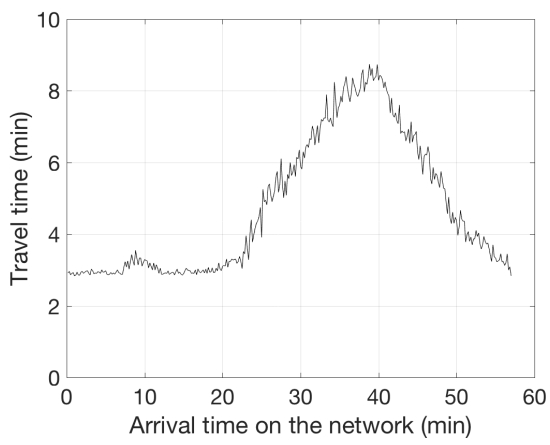

(d) travel times

FigURE 16: FOSIM observation models, seed 1 : cars only $(a$ and $c)$ and mixed traffic $(b$ and $d$ ) 
traffic model (without data assimilation) indicate that the corridor is freeflowing, with travel times stabilized around 3 mins (175s).

\subsection{Results with different observation models}

Three observation models have been tested : 'loop only', 'probe only', and 'combined loop and probe'. Travel time estimation based on the three observation models are illustrated in Figure 17, ground truth (in red) and default model travel times (in black) are also displayed.

During the free-flowing period, travel times are properly estimated regardless of observation models or traffic composition. However, significant differences are observed when a congestion occurs. The results analysis only focuses on the period $t=[20-45]$ min when the congestion is onset.

TSE based on the loop observation model underestimates travel times during the congestion period, regardless of traffic composition. This can be caused by an underestimation of the upstream demand and/or an overestimation of the supply. Loop 1 located at the entrance of the network is supposed to update the demand according to the ground truth, so the overestimation of the supply is the cause : loop 2 is located 100 meters upstream the head of the congestion, which cannot detect immediately after its onset.

TSE based on the probe observation model presents a better performance. However, it tends to overestimate travel times. It can be caused by a poor prior estimation of demand, which skews the n-index estimation of probe vehicle and leads to poor estimation of arrival/supply times. It can also be caused by poorly calibrated traffic parameters in the traffic model and/or non FIFO observations, which is confirmed in Figure 17(b) that shows the overestimation is enhanced for a mixed traffic (ranging from $t=[30-40] \mathrm{min}$ ). By analyzing FOSIM trajectories, it is observed that during congestion trucks are stuck on the right-most lane (over congested) while most of the cars travel faster on left-most lanes. The FIFO assumption is not fulfilled and the consequence on the performance of TSE can be explained as follows. When a probe vehicle (for instance a truck) returns its position, arrival and supply times are estimated in the (FIFO) mesoscopic model thereof. We conclude that when the characteristics of probe vehicles are distant from the mean traffic stream, this induces bias in traffic state estimation which tends to overestimate travel times. 


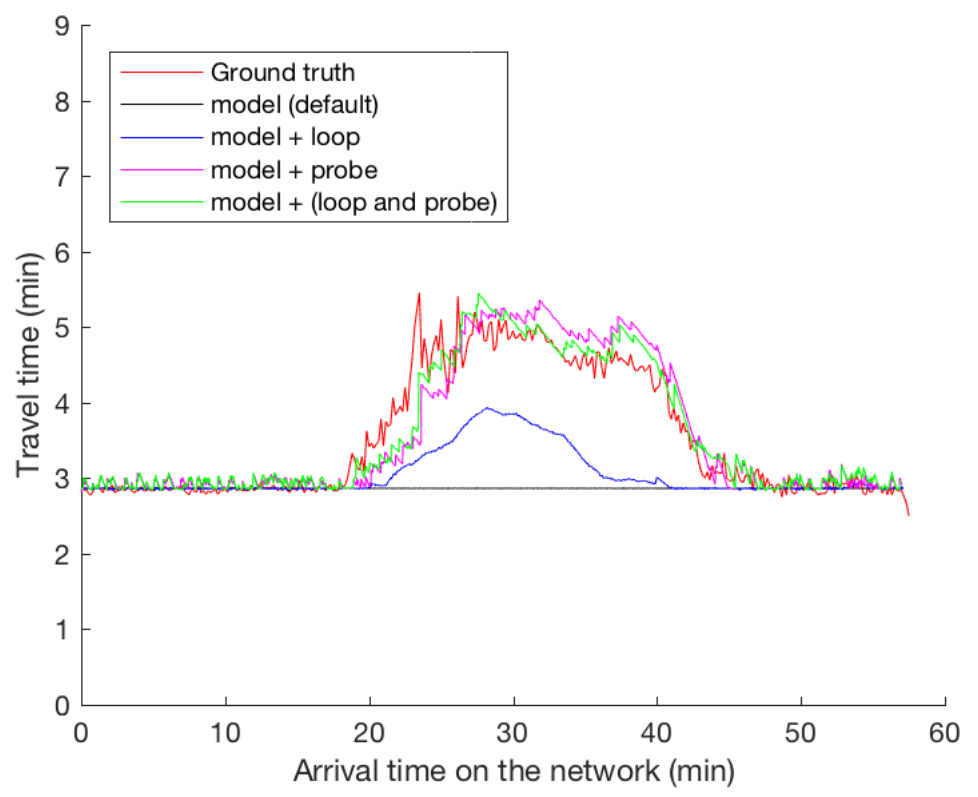

(a) Cars only, seed 1

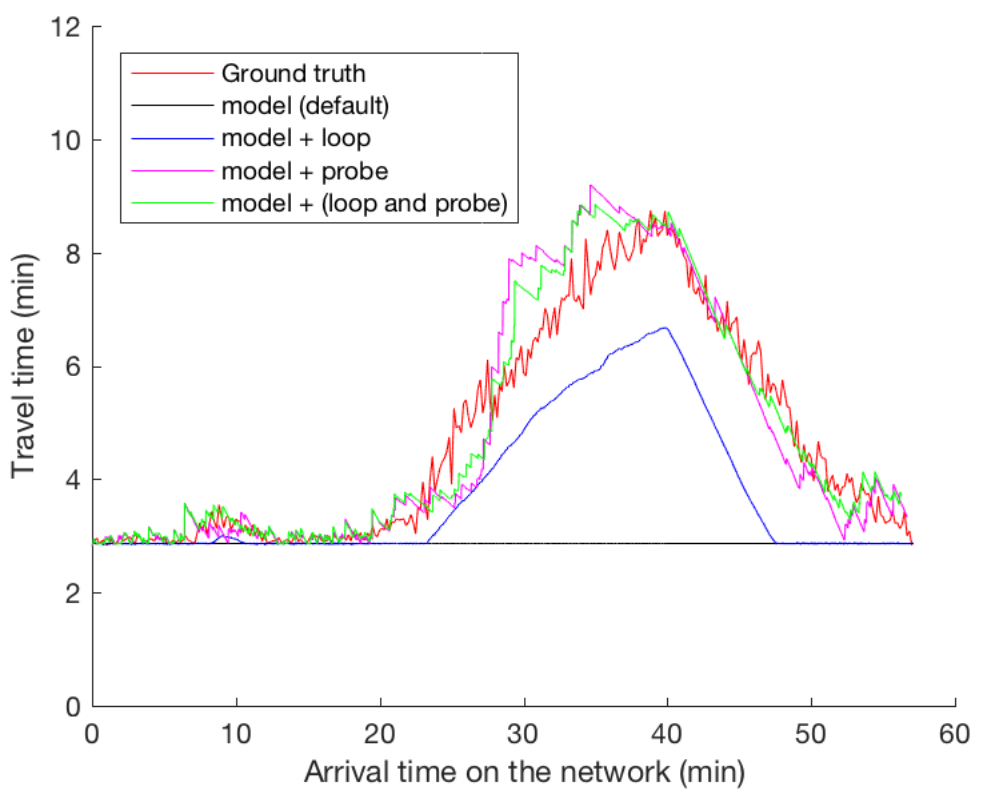

(b) Mixed traffic, seed 1

Figure 17: Comparison of estimated travel times 
TABLE 3: Scenario with cars only - performance of the different observational models (10 replications)

\begin{tabular}{|l|c|c|c|c|}
\hline & model & model + loop & model + probe & model + (loop and probe) \\
\hline RMSE $(s)$ & 72 & 57 & 25 & 25 \\
\hline MAPE $(\%)$ & 24 & 20 & 9 & 8 \\
\hline MPE $(\%)$ & -24 & -19 & 0.65 & 0.43 \\
\hline
\end{tabular}

As expected, TSE combining loop and probe observations outperforms the estimations considering loop and probe separately. The increase of travel times is detected immediately after the onset of congestion and the estimated travel times dynamically correspond to the ground truth.

Tables 3 and 4 provide three MoEs that have been calculated over the period $t=[20-45] \min$ : RMSE, MAPE and MPE. All the MoEs globally confirm the previous comments. In both scenarios, the estimation results with both data sources possess limited improvement compared to the probe-only cases. This can be explained by the fact that the demand input in the probeonly case well represents the actual demand so that the contribution from the loop data for flow correction is marginal. If the input demand contains noise, the improvement by assimilating additional loop data would be larger.

TABLE 4: Scenario with cars and trucks - performance of the different observational models (10 replications)

\begin{tabular}{|l|c|c|c|c|}
\hline & model & model + loop & model + probe & model + (loop and probe) \\
\hline RMSE $(s)$ & 234 & 117 & 112 & 109 \\
\hline MAPE $(\%)$ & 50 & 27 & 20 & 19 \\
\hline MPE $(\%)$ & -50 & -27 & 8 & 14 \\
\hline
\end{tabular}

5.4. Sensitivity to the penetration rate of probe vehicles

We expect that the performance of the data assimilation process can be improved when the amount of available data increase $(n-, x$ - and $t$ - frequency) and data are disaggregated. Here, we investigate the sensitivity of the proposed framework regarding the penetration rates of probe vehicles. The sensitivity has been tested on the scenario with cars only, with various penetration rates range from $0 \%$ to $10 \%$. Figure 18 illustrates the sensitivity of the method regarding the penetration rates of probe vehicles (over 


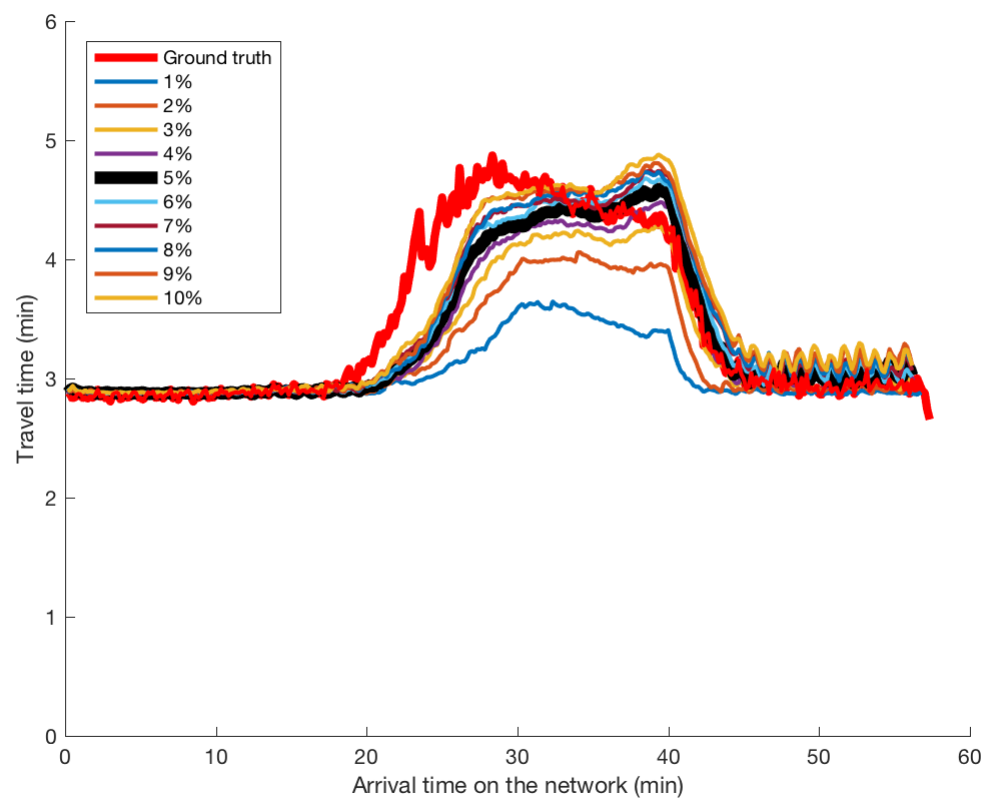

FiguRE 18: Travel time estimation for different probe penetration rates (10 replications)

10 replications). The estimation performance regarding MoEs is depicted in Figure 19.

The performance of the TSE is improved with only one percent of the observed probe vehicles. We also observe that the performance becomes marginal as the percentage of probe vehicle exceeds $6 \%$. It can be interpreted as follow : the gain for data assimilation is marginal and the remaining improvement needs to be found elsewhere, for instance, with the three other aspects : First, the model relies on assumptions and limitations (triangular fundamental diagram, FIFO traffic stream, etc.). Second, the definition of the demand on the network, which determines the traffic volumes that highly impacts the performance of the $n$-index estimation. And last but not least, the calibration of the model. We recall that the model has been poorly calibrated (default parameters) to emphasize the benefit of data assimilation procedure. Substantial gain is expected with a proper calibration procedure during the preparation of the model. We conclude that low penetration rates, around 5\%, are sufficient for a realistic traffic state estimation. 

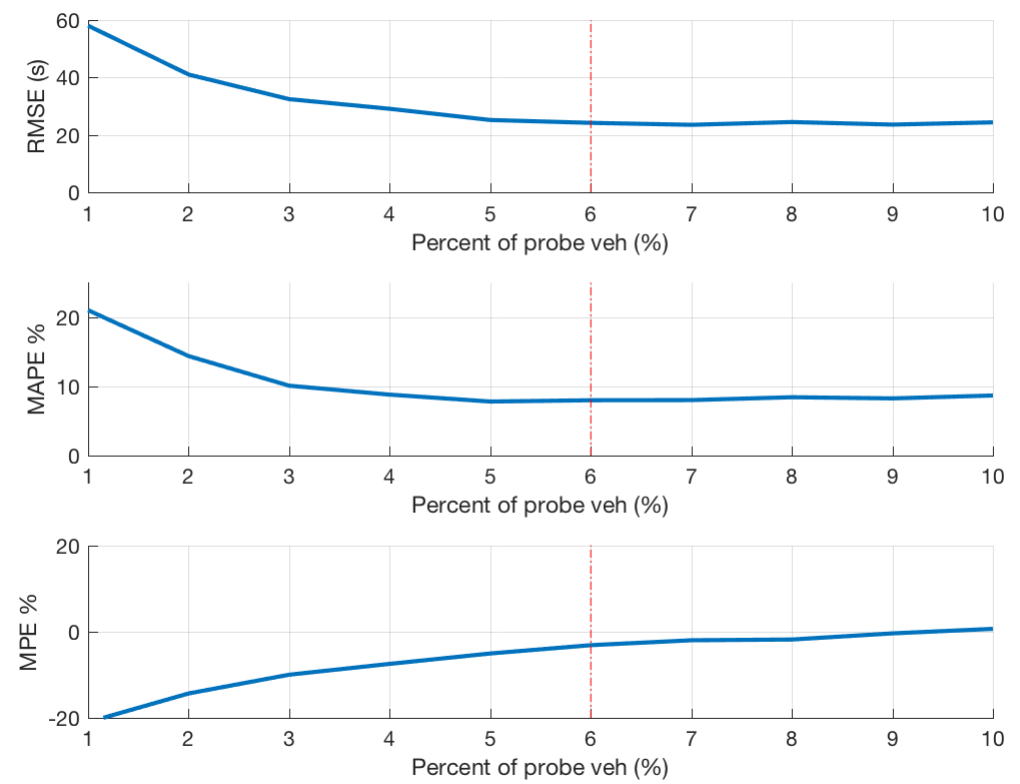

FiguRE 19: MoEs for different probe penetration rates (10 replications) 


\section{Discussion}

Based on the previous results, we conclude that Eulerian observations can update arrival and supply times, by adjusting the demand (flow) via adding or deleting vehicles locally. However, since loop sensors are spatially fixed, they only catch supply information as information propagates upstream. Hence, travel time estimation might be inconsistent (see section 4.2, the case of the downstream cell). It is therefore critical to locate loop sensors at the spots of a jam and a bottleneck to provide accurate estimation.

In contrast, Lagrangian observations spread over the network in space and time. Given a reasonable resolution (above 10\%), they can update both arrival times and supply times without any latency. However, two limitations exist in this method. First, the $n$-index estimation relies on the assumption of the FIFO condition, which is unrealistic. This might lead to poor estimation of travel times when probe information deviates from the average traffic conditions (see section 5.3). Second, the demand/flow at cell boundaries cannot be adjusted. A prior estimation of the demand will improve the performance of TSE with probe data (see section 4.2).

The combination of the two data sources compensates the limitations of each other. The experiment results demonstrate that TSE with data combination outperforms the estimation with a single source.

\section{Conclusion}

\subsection{Main findings}

A TSE estimator based Eulerian observations combined with a mesoscopic LWR model has been proposed and validated in [12]. This paper complements the methodology with Lagrangian observations. Now both Eulerian and Lagrangian observations can be used for TSE in a unique framework.

Eulerian observations provide comprehensive observations in time and vehicle for a discrete set of locations in the network. At those locations, model states are successfully revised, which provide good performance when observations are located near the head of congestion. The update acts as a 'flow regulator' at cell boundaries by adding, deleting, advancing or delaying vehicles.

Lagrangian observations provide a homogeneous coverage of the network in time and space for a discrete set of (probe) vehicles. Probe vehicles allow for an revision of demand-supply times at neighboring cell boundary of the 
network. The update acts as a cell 'travel time regulator' that yields good results under the condition that the demand on the network is known. Note that the $n$-index values of probe vehicles are critical and essential in the proposed approach. The calculation of this variable is application-specific (under FIFO or non-FIFO condition) regarding estimation performance, and it is subjective for further investigation.

In the TSE framework with data combination as proposed in section 3.3, Eulerian and Lagrangian observations become highly complementary. Eulerian observations successfully update traffic states (especially the flow) at loop locations of the network while Lagrangian observations successfully update cell travel times along the network. The estimation outperforms the scenarios considering loop and probe observations separately.

The methodology has been verified on the synthetic data derived from the same underlying traffic flow model. Meanwhile, the proposed TSE framework has been applied to a freeway corridor with a relaxed FIFO condition and distributed driving behavior. The validity has been tested using the data from a microscopic simulator, and the performance is satisfactory even for low rate of probe vehicles around 5\%. With increasing estimation accuracy and computational efficiency, the proposed TSE framework will be beneficial for decision support traffic management.

\subsection{Further research}

The robustness of data assimilation methodology is demonstrated in the paper. However, we propose here some avenues to improve the present methodology. On the model side, assumptions are to be relaxed to enhance the model and its ability to reproduce well-documented traffic flow phenomenons (non-FIFO condition, multi-class traffic, capacity drop). On the observation side, the exponential growth data sources (e.g. bluetooth - mobile - information from connected vehicles) will provide massive additional Lagrangian and Eulerian information, which can be assimilated based on the proposed framework providing their reliability and the proposition of adequate transformation operators.

The data assimilation framework represents a solid base for on-line estimating the reliability of both, again, traffic model and observed data. On the model side, during the assimilation procedure, the discrepancy between the background states from the model and the analysis states can be analyzed for detecting incident on road networks and adjusting the model (demand, parameters) accordingly. On the observed data side, the discrepancy between 
the observed states and the analysis states can be help for estimating the observation reliability or detecting a problem with the data collection system. And finally, we should recall that data assimilation has two main objectives : to provide a continuous estimation of traffic states at the present time based on discrete and aggregate observations; and to propose the most possible short term evolution of traffic states. The present paper is mainly focused on the first item. Its capability to provide robust short term forecasts should be explored, for instance, by implementing the proposed framework on a real large-scale network. 


\section{Acknowledgment}

${ }_{641}$ The authors sincerely thank L. Leclercq (University of Lyon, ENTPE, ${ }_{642}$ IFSTTAR, LICIT, UMR-T9401) for the fruitful discussions and assistance ${ }_{643}$ on the methodology presented in the paper. We would also like to thank 644 anonymous reviewers for their valuable suggestions. 
[1] Ansorge, R. : What does the entropy condition mean in traffic flow theory? Transportation Research Part B : Methodological 24(2), 133$143(1990)$

[2] Aubin, J.P., Bayen, A.M., Saint-Pierre, P. : Dirichlet problems for some hamilton-jacobi equations with inequality constraints. SIAM Journal on Control and Optimization 47(5), 2348-2380 (2008)

[3] Chu, L., Oh, S., Recker, W. : Adaptive kalman filter based freeway travel time estimation. In : Proceedings of the Transportation Research Board 84th Annual Meeting. Washington D.C. (2005)

[4] Clairais, A., Duret, A., El Faouzi, N.E. : Calibration of the fundamental diagram based on loop and probe data. Transportation Research Record : Journal of the Transportation Research Board (2560), 17-25 (2016)

[5] Claudel, C.G., Bayen, A.M. : Convex formulations of data assimilation problems for a class of hamilton-jacobi equations. SIAM Journal on Control and Optimization 49(2), 383-402 (2011)

[6] Courant, R., Friedrichs, K., Lewy, H. : On the partial difference equations of mathematical physics. IBM journal 11(2), 215-234 (1967)

[7] Daganzo, C.F. : A simple traffic analysis procedure. Networks and Spatial Economics 1(1-2), 77-101 (2001)

[8] Daganzo, C.F. : A variational formulation of kinematic waves : basic theory and complex boundary conditions. Transportation Research Part B : Methodological 39(2), 187-196 (2005)

[9] Deng, W., Lei, H., Zhou, X. : Traffic state estimation and uncertainty quantification based on heterogeneous data sources : A three detector approach. Transportation Research Part B : Methodological 57, 132-157 (2013)

[10] Dijker, T. : Fosim (freeway operations simulation) (2012). URL http ://www.fosim.nl

[11] Dimet, F.X.L., Talagrand, O. : Variational algorithms for analysis and assimilation of meteorological observations : theoretical aspects. Tellus A 38(2), 97-110 (1986) 
[12] Duret, A., Leclercq, L., El Faouzi, N.E. : Data assimilation using a mesoscopic lighthill-whitham-richards model and loop detector data. Transportation Research Record : Journal of the Transportation Research Board 2560, 26-35 (2016)

[13] Godunov, S.K. : A difference method for numerical calculation of discontinuous solutions of the equations of hydrodynamics. Matematicheskii Sbornik 47(89)(3), 271-306 (1959)

[14] Herrera, J.C., Bayen, A.M. : Incorporation of lagrangian measurements in freeway traffic state estimation. Transportation Research Part B : Methodological 44(4), 460-481 (2010)

[15] Kalnay, E. : Atmospheric modeling, data assimilation and predictability. Cambridge university press (2003)

[16] Kordic, V. : Kalman Filter. Intech, Vukovar, Croatia (2010)

[17] Laval, J., Leclercq, L. : The hamilton-jacobi partial differential equation and the three representations of traffic flow. Transportation Research Part B : Methodological 52, 17-30 (2013)

[18] Leclercq, L., Laval, J.A., Chevallier, E. : The lagrangian coordinates and what it means for first order traffic flow models. In : R. Allsop, M. Bell, B. Heydecker (eds.) Proceedings of the 17th International Symposium on Transportation and Traffic Theory, pp. 735-753. Elsevier, London, U.K. (2007)

[19] Lighthill, M., Whitham, G. : On kinematic waves ii : A theory of traffic flow on long crowded roads. Proceedings of Royal Society 229A(1178), 317-345 (1955)

[20] Nanthawichit, C., Nakatsuji, T., Suzuki, H. : Application of probevehicle data for real-time traffic-state estimation and short-term traveltime prediction on a freeway. Transportation Research Record 1855, 49-59 (2003)

[21] Newell, G. : A simplified theory of kinematic waves in highway traffic, part i : General theory. Transportation Research Part B : Methodological 27B(4), 281-287 (1993) 
[22] Ngoduy, D. : Applicable filtering framework for online multiclass freeway network estimation. Physica A : Statistical Mechanics and its Applications 387(2/3), 599-616 (2008)

[23] Payne, H. : Models of freeway traffic and control. Simulation Councils Proceedings Series : Mathematical Models of Public Systems 1(1), 51-61 (1971)

[24] Richards, P. : Shock waves on the highway. Operations Research 4(1), 42-51 (1956)

[25] Seo, T., Kusakabe, T. : Probe vehicle-based traffic state estimation method with spacing information and conservation law. Transportation Research Part C : Emerging Technologies 59, 391-403 (2015)

[26] Shannon, C.E. : Weaver; w.(1949) : The mathematical theory of communication. Urbana (1949)

[27] Talagrand, O. : Assimilation of observations, an introduction. JournalMeteorological Society of Japan Series 2 75, 81-99 (1997)

[28] Velan, S., Florian, M. : A note on the entropy solutions of the hydrodynamic model of traffic flow. Transportation Science 36(4), 435-446 (2002)

[29] Wang, Y., Papageorgiou, M. : Real-time freeway traffic state estimation based on extended kalman filter : A general approach. Transportation Research Part B : Methodological 39(2), 141-167 (2005)

[30] Work, D., Tossavainen, O.P., Blandin, S., Bayen, A., Iwuchukwu, T., Tracton, K. : An ensemble kalman filtering approach to highway traffic estimation using gps enabled mobile devices. In : Proceedings of the 47th IEEE Conference on Decision and Control, pp. 2141-2147. Cancun, Mexico (2008)

[31] Yuan, Y., Duret, A., Van Lint, H. : Mesoscopic traffic state estimation based on a variational formulation of the lwr model in lagrangian-space coordinates and kalman filter. Transportation Research Procedia 10, 82-92 (2015) 
[32] Yuan, Y., Van Lint, J.W.C., Wilson, R.E., Van Wageningen-Kessels, F.L.M., Hoogendoorn, S.P. : Real-time lagrangian traffic state estimator for freeways. IEEE Transactions on Intelligent Transportation Systems 13(1), 59-70 (2012) 